\title{
Propiedades psicométricas de la escala de competencia intrasexual a nivel intergrupal en Costa Rica
}

\author{
Psychometric Properties of the Intergroup-Intrasexual Competition \\ Scale in Costa Rica \\ Propriedades psicométricas da escala de competência intrasexual \\ ao nível intergrupal na costa rica
}

\author{
David Torres Fernández*, Vanessa Smith-Castro**, Abraham P. Buunk*** \\ ${ }^{*}$ Universidad de Costa Rica, *Universidad de Iberoamérica, ${ }^{* *}$ Universidad de Costa Rica \\ ${ }^{* * *}$ Universidad of Groningen y Academia Holandesa Real de las Ciencias
}

Doi: http://dx.doi.org/10.12804/revistas.urosario.edu.co/apl/a.3536

\section{Resumen}

Con el fin de proporcionar evidencias de validez de constructo para la escala de competencia intrasexual-intergrupal, diseñada por Klavina, Buunk y Park (2009), en la población costarricense, se realizaron dos estudios psicométricos con estudiantes universitarios entre los 16 y 31 años (204 estudiantes, $45,7 \%$ mujeres, en el primer estudio y 335 estudiantes, $50,4 \%$ mujeres, en el segundo). En estos estudios se aplicó un cuestionario que contenía la medida competencia intrasexual-intergrupal junto con otras mediciones de variables psicosociales y evolucionistas (competencia intrasexual, orientación a la dominancia social, orientación sociosexual, contacto intergrupal, control inhibitorio, valor como pareja). En dichos estudios se realizaron análisis de confiabilidad, análisis factoriales exploratorios y confirmatorios, y análisis de correlaciones. Los resultados mostraron que la escala puede ser considerada como una medida unidimensional y que sus puntuaciones correlacionan de forma positiva con medidas de competencia intrasexual, orientación sociosexual y orientación a la dominancia social; y de forma negativa con medidas contacto intergrupal, control inhibitorio y de valor como pareja, tal y como se esperaba según la evidencia empírica. Se discute el aporte de esta medida al estudio del comportamiento humano desde la psicología social y la psicología evolucionista.

Palabras clave: selección de pareja, competencia intrasexual, relaciones intergrupales, psicología evolucionista.

* Universidad de Costa Rica, Universidad de Iberoamérica, San José, Costa Rica. Correo electrónico: david.torresfernandez@ucr. ac.cr

** Universidad de Costa Rica, San José, Costa Rica. Correo electrónico: vanessa.smith@ucr.ac.cr

*** Universidad of Groningen, Groningen, Holanda. Academia Holandesa Real de las Ciencias, Ámsterdam, Holanda Correo electrónico: a.p.buunk@rug.nl

Cómo citar este artículo: Torres, F. D., Smith-Castro, V., \& Buunk, A. P. (2017). Propiedades psicométricas de la escala de competencia intrasexual a nivel intergrupal en Costa Rica. Avances en Psicología Latinoamericana, 35(2), 407-424. doi: http:// dx.doi.org/10.12804/revistas.urosario.edu.co/apl/a.3536 


\section{fibstract}

Two studies were conducted to provide evidence of construct validity of the Intergroup-Intrasexual Competition Scale, developed by Klavina, Buunk y Park (2009), among Costa Rican university students between 17 and 31 years old (204 students, $45.7 \%$ female, in the first study and 335 students, $50.4 \%$ female, in the second), measures of Intergroup Intrasexual Competition were applied with other measures of psychosocial and evolutionary variables (Intrasexual Competition, Social Dominance Orientation, Sociosexual Orientation, Intergroup Contact, Inhibitory Control, Mate Value). Results of both studies showed that the scale can be considered as a measure of a one-dimension construct, correlating positively with measures of Intrasexual Competition, Sociosexual Orientation and Social Dominance Orientation, and negatively with measures of Mate Value, Perceived Contact Quality and Inhibitory Control, as predicted by theory and previous empirical data. The utility of this measure in the study of human behavior from social and evolutionary psychology is discussed.

Keywords: mate selection, intrasexual competition, intergroup relations, evolutionary psychology.

\section{Resumo}

Com o fim de proporcionar evidências de validez de constructo para a Escala de Competência intrasexual-Intergrupal, desenhada por Klavina, Buunk e Park (2009), em população costa-ricense se realizaram dois estudos psicométricos com estudantes universitários entre os 16 e 31 anos (204 estudantes, $45,7 \%$ mulheres, no primeiro estudo e 335 estudantes, 50,4\% mulheres no segundo) nos quais aplicou-se um questionário que continha a medida Competência Intrasexual-Intergrupal junto com outras medições de variáveis psicossociais e evolucionistas (Competência intrasexual, Orientação à Dominância Social, Orientação Sociossexual, Contato intergrupal. Controle Inibitório, Valor como Casal). Em ditos estudos se realizaram análises de confiabilidade, análises fatoriais, exploratórios e confirmatórios e, análises de correlações. Os resultados mostraram que a escala pode ser considerada como uma medida unidimensional e que as suas pontuações correlacionam de forma positiva com medidas de competência intrasexual, Orientação Sociossexual e Orientação à Dominância Social; e de forma negativa com medidas Contato intergrupal, Controle inibitório e de Valor como Casal, tal e como se esperava segundo a evidência empírica. Discute-se o aporte desta medida ao estudo do comportamento humano desde a psicologia social e a psicologia evolucionista.

Palavras-chave: Seleção de casal, Competência intrasexual, Relações intergrupais, Psicologia evolucionista.

La rivalidad intrasexual a la hora de seleccionar parejas del sexo opuesto ha sido ampliamente estudiada, lo cual ha proporcionado un importante conocimiento sobre las formas en que hombres y mujeres se relacionan entre sí en nuestra especie (Buss, 2006; Buunk, Massar \& Dijkstra, 2007; Buunk, Park \& Duncan, 2010; Fisher, Tran \& Voracek, 2008; Li et al., 2009). Sin embargo, en lo que respecta al contexto de las relaciones intergrupales o interétnicas, su investigación ha sido muy escasa (Klavina, Buunk \& Park, 2009), lo cual resulta sorprendente, ya que la rivalidad intergrupal probablemente ha sido constante en la evolución del ser humano. Así, el estudio de la rivalidad intrasexual en el contexto de las relaciones intergrupales resultaría de gran utilidad para comprender mejor las formas de expresión de la hostilidad entre grupos humanos, como el prejuicio y los estereotipos, así como sus últimas motivaciones.

La rivalidad intrasexual puede definirse como la competencia entre personas del mismo sexo para acceder a los miembros más deseables del sexo opuesto. Este tipo de competencia no emerge exclusivamente ante una confrontación directa ni involucra conductas diseñadas para obtener mejores recursos a expensas de quienes intentan 
adquirir dichos recursos. Los competidores no necesitan ni siquiera conocerse para ser rivales y esta competencia puede centrarse en la habilidad para localizar posibles parejas, la señalización del interés o disponibilidad para el cortejo, la adquisición de territorios o la alteración de la apariencia propia (Buunk \& Fisher, 1999).

Desde una perspectiva evolucionista, un exogrupo como los inmigrantes puede significar una amenaza para la población nacional, ya que invaden su territorio y el acceso a otros recursos como el alimento y las oportunidades de apareamiento dentro de su propio grupo interno (Sidanius \& Pratto, 1999). Ahora bien, la percepción de amenaza puede ser más o menos fuerte dependiendo del grupo de inmigrantes y del tipo de relaciones históricas con los nacionales. Los grupos de mayor estatus social serán considerados como más amenazantes debido a las jerarquías sociales que posicionan a ciertos grupos por encima del resto (Morrison, Fast \& Ybarra, 2009; Oldmeadow \& Fiske, 2007) y a las características del contacto con estos grupos en el marco de las relaciones intergrupales generales (Allport, 1954; Pettigrew, 1988). La percepción de las amenazas varía también en función de las diferencias individuales. Por ejemplo, los inmigrantes pueden resultar particularmente amenazantes para aquellos individuos con altos niveles de orientación a la dominancia social (SDO), es decir, con altas tendencias a legitimar las jerarquías sociales (Sidanius \& Pratto, 1999).

En general, la amenaza podría provocar celos y rivalidad, que serían más elevados si los rasgos del exogrupo son considerados más atractivos para el sexo opuesto y en función de las diferencias individuales en el valor como pareja, esto es, en las características que las personas perciben como deseables en sí mismos y en los otros para el apareamiento y la reproducción (Kirsner, Figueredo \& Jacobs, 2003). Así, las amenazas pueden ser mayores si se tiene una pobre autoestima en lo que respecta a la capacidad de competir intrasexualmente.
Un ejemplo de las pocas investigaciones sobre rivalidad intrasexual a nivel intergrupal en humanos es el trabajo de Klavina, Buunk y Park (2009), quienes estudiaron los niveles de celos de participantes holandeses con respecto a potenciales rivales de diferentes países, lo que busca definir cuáles características de estos grupos étnicos pueden predecir los celos evocados. Los autores encontraron, de forma general, que los grupos más amenazantes son aquellos que se perciben con una mayor inclinación a las relaciones a corto plazo y de mayor estatus social.

En los momentos históricos actuales de gran movilidad humana, se vuelve relevante la investigación de la competencia intrasexual a nivel intergrupal, tomando en cuenta también el nivel y la calidad de contacto con miembros del exogrupo como un factor influyente en las actitudes intergrupales (Allport, 1954; Johnson \& Jacobson, 2005; Pettigrew, 1988), las diferencias individuales en la legitimación de las desigualdades sociales (Sidanius \& Pratto, 1999) y el valor como pareja de sí mismo y de los otros en relaciones a corto y largo plazo (Kirsner, Figueredo \& Jacobs, 2003).

Un aporte central de la investigación de Klavina, Buunk y Park (2009) fue el desarrollo de la escala de competencia intrasexual a nivel intergrupal (ECInI), la cual fue diseñada para medir las actitudes de los estudiantes universitarios hacia el apareamiento intergrupal (con personas de diferentes nacionalidades o diferentes grupos etnoculturales). Dicha escala consta de doce ítems que miden actitudes hacia la selección de pareja intergrupal como "Los hombres y las mujeres de diferentes nacionalidades tienen muchas diferencias culturales como para casarse entre ellos" o "Si una mujer está siendo cortejada por varios hombres, ella debería darle prioridad al pretendiente que sea de su propio país". La escala posee una versión espejo para hombres y mujeres "Me dan ganas de darles su merecido a los hombres extranjeros que vienen aquí como turistas a seducir a nuestras mujeres" frente a "Me dan ganas de 
darles su merecido a las mujeres extranjeras que vienen aquí como turistas a seducir a nuestros hombres" (Klavina \& Buunk, 2013).

En su desarrollo, la versión original presentó altos niveles de consistencia interna en el contexto europeo $(\alpha=0,95)$ tanto en la muestra total, como cuando se separó por sexo $(\alpha=0,94$ para hombres, y $\alpha=0,81$ para mujeres). Dicha escala fue probada en 125 estudiantes universitarios holandeses (40 mujeres) con una edad media de 21,88 años (DE =2.64). Así mismo, los datos mostraron que esta escala tiene un comportamiento diferenciado por el sexo del participante, donde los hombres tienden a puntuar más alto que las mujeres. Los resultados de este estudio evidenciaron que altos puntajes en la escala se relacionan con una mayor derogación de los exogrupos en cortejo a corto plazo en los hombres; mientras que en las mujeres no se encontraron relaciones estadísticamente significativas entre ambos aspectos (Klavina, Buunk \& Park, 2009). Por el momento, no se han encontrado datos de adaptaciones $u$ otras versiones de esta escala en el ámbito mundial. El medir las propiedades psicométricas de una versión en español de esta escala en un contexto latinoamericano es el objetivo de los estudios aquí presentados.

\section{Contexto de la presente investigación}

En Costa Rica, para el año 2011 habitaban 4.301712 personas, de las cuales, 385889 eran extranjeros (Instituto Nacional de Estadísticas y Censos, 2011). De acuerdo con esto, la cantidad de extranjeros que viven en Costa Rica ha aumentado en un $526 \%$ en 28 años, una cantidad importante si se tiene en cuenta que el aumento total de la población ha sido del 78\% (61663 extranjeros y 2416809 nacionales en el censo de población de 1984). Este aumento afecta sin duda la manera en que los costarricenses han visto influenciados varios aspectos de sus vidas por la presencia de extranjeros, especialmente de nicaragüenses, quienes representan el 74,6\% de los inmigrantes en el país y quienes son vistos como una amenaza a la seguridad social y a las oportunidades de trabajo debido a su baja condición social (Sandoval, 2002; Smith-Castro, Araya \& Peña, 2009). Además de la población migrante nicaragüense, en Costa Rica conviven otros grupos de inmigrantes importantes, como colombianos, estadounidenses, italianos, peruanos, salvadoreños, españoles y cubanos.

La presente investigación se llevó a cabo en la provincia de Limón en Costa Rica. Esta provincia se caracteriza por ser una región de alta diversidad cultural, con una fuerte presencia de afrocostarricenses, chinocostarricenses, indígenas, europeos, estadounidenses, y otros grupos étnicos nacionales y extranjeros (Instituto Nacional de Estadísticas y Censos, 2011). Se trata de un contexto social ideal para estudiar la disposición hacia la rivalidad intrasexual en un contexto intergrupal, debido a la gran presencia de exogrupos claramente diferenciados.

En resumen, el objetivo del presente artículo es presentar evidencias de validez y confiabilidad de la escala de competencia intrasexual a nivel intergrupal (ECInI) en un contexto intercultural latinoamericano por primera vez. Para esto se llevaron a cabo dos estudios, en los cuales se contrastaron las siguientes hipótesis con el fin de encontrar evidencias de validez discriminante y concurrente de la escala:

(I) Al igual que en su versión original, se encontrarán mayores niveles de rivalidad intrasexual-intergrupal en los hombres que en las mujeres.

(II) De igual forma, se hipotetiza una relación positiva entre la rivalidad intrasexual general y la rivalidad intrasexual específica que mide esta escala (en el nivel intergrupal) y la orientación sociosexual (diferencias individuales en permisividad sexual y promiscuidad, Penke, 2010). Por otro lado, dada la relación existente entre las actitudes hacia la rivalidad intrasexual a nivel intergrupal y la orientación a la dominancia social, se espera 
encontrar una relación positiva entre medidas de estos dos constructos.

(III) En concordancia con la extensa investigación sobre contacto intergrupal (Allport, 1954, Pettigrew 1998), se espera una relación negativa entre ambas variables, de forma tal que a mayor calidad percibida de contacto intergrupal, menor será la disposición hacia la rivalidad intrasexual con el exogrupo. De igual forma, se espera una correlación negativa entre puntuaciones en medidas de control inhibitorio y la rivalidad intrasexual a nivel intergrupal, considerando la extensa literatura que indica que una mayor auto-regulación general regula la manifestación de estereotipos y conductas discriminatorias (Amodio \& Devine, 2010; Plant \& Devine, 2009), lo cual podría extenderse a las percepciones de amenaza en un ambiente de competencia intrasexual. Finalmente, se esperan relaciones negativas entre la escala de competencia intrasexual a nivel intergrupal con medidas del propio valor como pareja y del valor asignado a potenciales parejas, ya que valores de pareja bajos indicarían una mayor inseguridad en la capacidad de conseguir parejas deseables, lo cual limita las exigencias en la selección de pareja, lo que a su vez provocaría mayores celos y rivalidad ante extranjeros (Kirsner, Figueredo \& Jacobs, 2003).

\section{Estudio 1. Exploración de las propiedades psicométricas de la escala}

\section{Método}

Participantes. Se tuvo en cuenta que las recomendaciones sobre el tamaño de una muestra en estudios psicométricos varían dependiendo del tipo y características del instrumento que se desea probar — dependiendo de sus finalidades, consecuencias y técnicas de análisis a utilizar para recolectar las evidencias de validez y confiabilidad-; de esta manera, se tiene que los tamaños de muestra de
100 personas son adecuados para considerar la consistencia interna de un instrumento (Sheng \& Sheng, 2012), los de 150 personas son suficientes para obtener soluciones satisfactorias en análisis factoriales exploratorios (Guadagnoli \& Velicer, 1988) y las muestras mayores a 200 personas son adecuadas para estimar modelos de ecuaciones estructurales (Hoe, 2008). Por lo anterior, los datos del Estudio 1 fueron recopilados en una muestra de 204 estudiantes universitarios que participaron de forma voluntaria en el estudio. Las edades de los participantes oscilaban entre los 17 y 31 años $(\mathrm{M}=22,34, \mathrm{DE}=3,06)$. Un 45,7\% de las participantes eran mujeres. Todos los participantes eran estudiantes de la sede de Limón de la Universidad de Costa Rica.

Instrumentos. El cuestionario incluía un módulo de preguntas sociodemográficas, la ECInI (Klavina, Buunk \& Park, 2009) y tres escalas de validación: la escala de competencia intrasexual (Buunk \& Fisher, 2009), la escala de orientación sociosexual (Penke, 2010) y medidas de valor como pareja (Kirsner, Figueredo \& Jacobs, 2003).

La ECInI posee una versión para hombres y otra para mujeres y consiste de doce ítems que se califican en una escala Likert de 1 (totalmente en desacuerdo) a 7 (totalmente de acuerdo). En virtud de que el eje central del presente trabajo es estudiar las propiedades psicométricas de la escala, estos resultados se presentarán más adelante.

La escala de competencia intrasexual (Buunk \& Fisher, 2009) también posee una versión para hombres y otra para mujeres y, de igual forma, los reactivos se califican en una escala Likert de 1 (totalmente en desacuerdo) a 7 (totalmente de acuerdo). Consta de doce ítems y presenta una alta consistencia interna $(\alpha=0,86)$.

En la escala de orientación sociosexual (Penke, 2010) los reactivos se califican en una escala de 1 a 9. Cada subescala consta de tres ítems y poseen una consistencia interna adecuada para fines de investigación ( $\alpha=0,89$ conductual, $\alpha=0,69$ actitud y $\alpha=0,87$ deseo). 
Las medidas de valor como pareja evalúan características deseables en potenciales parejas a corto y largo plazo y una autovaloración de dichas características. Las tres escalas son parte de The Mate Value Inventory (Kirsner, Figueredo \& Jacobs, 2003) y están conformadas por una lista de adjetivos en las cuales el sujeto debe de asignar un valor de 0 a 10 de acuerdo con si el aspecto es necesario o no en una potencial pareja. La consistencia interna de cada escala es aceptable $(\alpha=$ $0,88, \alpha=0,76$ y $\alpha=0,76$, respectivamente).

Procedimientos. Se contactaron a los estudiantes en sus respectivas aulas universitarias en el Recinto de Limón de la Universidad de Costa Rica, C. R. Se les explicó el objetivo de la investigación y se les solicitó llenar voluntariamente el cuestionario. En la portada del cuestionario aparecía el respectivo consentimiento informado, siguiendo los lineamientos del Comité Ético Científico de la Universidad de Costa Rica. Los y las estudiantes expresaron su acuerdo en participar en la investigación devolviendo el cuestionario completo. Completarlo les tomó aproximadamente $10 \mathrm{mi}$ nutos. Los análisis se realizaron con los paquetes estadísticos SPSS 16.0.

\section{Resultados}

Estructura. Un análisis de factores exploratorio mediante factorización de ejes y rotación Varimax (tabla 1) evidenció la idoneidad de muestral para llevar a cabo el análisis $(\mathrm{KMO}=0,891$, Bartlett Chi-Cuadrado $\left(\chi^{2}\right)=1818,79, \mathrm{gl}=66, \mathrm{p}<0,001$. Los resultados indican la presencia de dos factores con valores característicos mayores a uno, que en su conjunto explicaron un $69,41 \%$ de la varianza total. El primer factor, compuesto por siete ítems, presentó un valor característico de 7,12 y explicó un 59,36\% de la varianza. Los reactivos que componían este factor reflejan las reacciones típicas ante la que representan miembros de exogrupo como celos, rivalidad y necesidad de confrontación ("Me resulta repugnante ver a los hombres extranjeros seduciendo a mujeres costarricenses) y consecuentemente fue denominado "amenaza". El segundo factor, compuesto por cinco ítems presentó un valor característico de 1,20 y explicó un $10,04 \%$ de la varianza (tabla 1). Los ítems que componían este factor expresaban la desaprobación social y las consecuencias negativas para la sociedad si se promueven las relaciones entre nacionales y extranjeros ("Las personas que se casan con extranjeros son responsables por la disminución de la población costarricense") y, por lo tanto, se denominó "desaprobación normativa". Ahora bien, es importante hacer notar que el primer factor explica por sí solo el $60 \%$ de la varianza de los reactivos, donde se observa una importante caída en el valor característico del primer al segundo factor, y que una inspección de la matriz de componentes no rotados evidenció que todos los ítems presentaron cargas factoriales altas en el primer factor previo a la rotación. Todo eso hace pensar que los reactivos se inclinan a covariar en una sola escala unidimensional.

Consistencia interna. Dados los resultados del análisis exploratorio de factores, se llevó a cabo un análisis de consistencia interna mediante Alfa de Cronbach para cada uno de los factores y para la escala global. El primer factor, amenaza, presentó una adecuada consistencia interna a nivel general $(\alpha=0,91)$ y por separado, para hombres $(\alpha=0,88)$ y mujeres $(\alpha=0,93)$, respectivamente. De igual forma, el factor de desaprobación normativa mostró una adecuada consistencia interna a nivel general $(\alpha=0,88)$ y para hombres $(\alpha=0,89)$ y mujeres $(\alpha=0,87)$, respectivamente. La escala completa presentó también una alta consistencia interna a nivel general $(\alpha=0,93)$ y al separarla por sexo $(\alpha=0,94$ para las mujeres y $\alpha=0,92$ para los hombres). En todos los casos anteriores, las correlaciones ítem-total de los reactivos obtuvieron valores mayores a 0,44 .

Diferencias por sexo. Si se considera el rango teórico de la escala de 1 a 7 (dadas las opciones de respuesta), la escala general presentó una puntaje bajo $(\mathrm{M}=1,68, \mathrm{DE}=1,09)$ con valores entre $1 \mathrm{y}$ 
Tabla 1

Resumen los resultados del análisis exploratorio de factores de la ECInI en el Estudio $1(N=204)$

\begin{tabular}{|c|c|c|c|}
\hline & \multicolumn{2}{|c|}{ Cargas factoriales } & \\
\hline & 1 & 2 & \\
\hline Valor característico & 6,79 & 0,85 & \\
\hline$\%$ Varianza explicada & 56,58 & 7,10 & \\
\hline Reactivos & & & Comunalidad \\
\hline $\begin{array}{l}\text { Los hombres y las mujeres de diferentes nacionalidades tienen muchas diferencias } \\
\text { culturales como para casarse entre ellos. }\end{array}$ & & 0,594 & 0,423 \\
\hline $\begin{array}{l}\text { Las personas que se casan con extranjeros son responsables por la disminución de } \\
\text { la población costarricense. }\end{array}$ & & 0,807 & 0,751 \\
\hline $\begin{array}{l}\text { Si una mujer está siendo cortejada por varios hombres, ella debería darle prioridad } \\
\text { al pretendiente que sea de su propio país. }\end{array}$ & & 0,729 & 0,663 \\
\hline $\begin{array}{l}\text { Las mujeres costarricenses deberían esforzarse por atraer a los hombres costarri- } \\
\text { censes, de lo contrario las mujeres extranjeras los atraerán y se casarán con ellos. }\end{array}$ & & 0,767 & 0,624 \\
\hline $\begin{array}{l}\text { Los hombres extranjeros que seducen a las mujeres costarricenses lo hacen para } \\
\text { humillar a los hombres costarricenses. }\end{array}$ & & 0,611 & 0,624 \\
\hline Me enoja ver que tantas mujeres costarricenses se estén casando con extranjeros. & 0,580 & & 0,626 \\
\hline $\begin{array}{l}\text { Cuando los extranjeros vienen a nuestro país a seducir a nuestras mujeres, me dan } \\
\text { ganas de interferir. }\end{array}$ & 0,645 & & 0,721 \\
\hline $\begin{array}{l}\text { Me resulta repugnante ver a los hombres extranjeros seduciendo a mujeres costarri- } \\
\text { censes. }\end{array}$ & 0,595 & & 0,536 \\
\hline A mis padres no les gustaría que yo me casara con una extranjera. & 0,567 & & 0,344 \\
\hline $\begin{array}{l}\text { Me molesta que una familiar cercana (mi hermana o mi prima) salga con alguien de } \\
\text { otro país. }\end{array}$ & 0,860 & & 0,848 \\
\hline $\begin{array}{l}\text { Me molesta que un familiar cercano (mi hermano o mi primo) salga con alguien de } \\
\text { otro país. }\end{array}$ & 0,880 & & 0,854 \\
\hline $\begin{array}{l}\text { Me dan ganas de darles su merecido a los hombres extranjeros que vienen aquí co- } \\
\text { mo turistas a seducir a nuestras mujeres. }\end{array}$ & 0,636 & & 0,628 \\
\hline
\end{tabular}

Nota. Factor 1 = Amenaza, Factor 2 = Desaprobación Normativa. Método de extracción = Factorización de Ejes. Método de Rotación = Varimax con Kaiser. Se muestran solamente las cargas factoriales mayores a 0,40.

6,50. Para determinar potenciales diferencias entre ambos sexos en la ECInI, se realizó una prueba $t$ para muestras independientes para los valores medios de la escala completa y sus subescalas. Las diferencias en los niveles de rivalidad expresadas ante los miembros del exogrupo no fueron significativas $\left(t_{199}=0,474, p=0,64, d=0,07\right)$. Tampoco se encontraron diferencias significativas al realizar los análisis para cada una de las subescalas $\left(_{199}=\right.$
$0,921, p=0,36, d=0,12$ para el de amenaza y $t_{199}$ $=-0,242, p=0,81, d=0,03$ para el de desaprobación normativa) (ver tabla 2 ).

Evidencias de validez. La matriz de correlaciones simples (tabla 2) evidenció correlaciones significativas y positivas entre las puntuaciones de la ECInI total y las puntuaciones en la medida competencia intrasexual general $(r=0,53, p<$ 
$0,001)$ y el aspecto conductual de la orientación sociosexual ( $r=0,15, p=0,036)$. Por otro lado, se observaron correlaciones significativas y negativas con los valores asignados a potenciales parejas a corto $(r=-0,16, p=0,025)$ y largo plazo $(r=-0,16, p=0,020)$.

En el caso de los hombres, se observó una correlación positiva significativa de la ECInI con la competencia intrasexual general $(r=0,51, p<$ $0,001)$ y correlaciones negativas con el valor autoasignado como pareja $(r=-0,22, p=0,025)$ y el valor asignado a potenciales parejas a corto plazo $(r=-0,20, p=0,043)$. En el caso de las mujeres, se encontraron correlaciones positivas y significativas entre las puntuaciones de la ECInI y las puntuaciones en competencia intrasexual general $(r=0,55, p<0,001)$ y los aspectos conductuales $(r=0,37, p<0,001)$ y de deseo $(r=0,34, p=$ $0,001)$ de la orientación sociosexual.
La dimensión de amenaza presentó una correlación positiva con la competencia intrasexual general $(r=0,50, p<0,001)$, así como correlaciones negativas con el valor asignado a sí mismo como pareja $(r=-0,15, p=0,034)$ y el valor asignado a potenciales parejas a corto plazo $(r=$ $-0,24, p=0,001)$.

En el caso de los hombres, se encontró una correlación positiva y significativa entre la amenaza y la competencia intrasexual $(r=0,50, p<$ $0,001)$, así como una correlación negativa con el valor autoasignado como pareja $(r=-0,27, p=$ $0,004)$. En el caso de las mujeres, se encontraron relaciones positivas entre la amenaza y la competencia intrasexual general $(r=0,51, p<0,001)$ y el aspecto conductual de la orientación sociosexual $(r=0,27, p=0,009)$.

La dimensión de desaprobación normativa correlacionó positivamente con la competencia intra-

Tabla 2

Medias, desviaciones estándar, Alfas de Cronbach de la ECInI y sus correlaciones bivariadas con las escalas de validación en el Estudio $1(N=204)$

\begin{tabular}{|c|c|c|c|c|c|c|c|c|c|c|}
\hline & m & SD & $\alpha$ & $\begin{array}{c}\text { Compet. } \\
\text { Intrasexual }\end{array}$ & $\begin{array}{c}\text { Auto } \\
\text { valor de } \\
\text { pareja }\end{array}$ & $\begin{array}{l}\text { Valor pa- } \\
\text { reja corto } \\
\text { plazo }\end{array}$ & $\begin{array}{l}\text { Valor pa- } \\
\text { reja largo } \\
\text { plazo }\end{array}$ & $\begin{array}{c}\text { OS } \\
\text { (conducta) }\end{array}$ & $\begin{array}{c}\text { OS } \\
\text { (actitud) }\end{array}$ & $\begin{array}{c}\text { OS } \\
\text { (deseo) }\end{array}$ \\
\hline \multicolumn{11}{|l|}{ Ambos sexos } \\
\hline ECInI & 1,68 & 1,09 & 0,91 & $0,53^{* *}$ & $-0,12$ & $-0,16$ & $-0,16^{* *}$ & $0,15^{*}$ & 0,04 & 0,13 \\
\hline Amenaza & 1,80 & 1,25 & 0,93 & $0,50^{* *}$ & $-0,15^{*}$ & $-0,24^{* *}$ & $-0,11$ & 0,12 & $-0,07$ & 0,02 \\
\hline Desaprobación & 1,60 & 1,13 & 0,88 & $0,47^{* *}$ & $-0,09$ & $-0,08$ & $-0,18^{* *}$ & $0,15^{*}$ & $-0,01$ & $0,21^{* *}$ \\
\hline \multicolumn{11}{|l|}{ Hombres } \\
\hline ECInI & 1,65 & 1,06 & 0,92 & $0,51^{* *}$ & $-0,22^{*}$ & $-0,20^{*}$ & $-0,18$ & 0,00 & $-0,12$ & $-0,03$ \\
\hline Amenaza & 1,82 & 1,30 & 0,88 & $0,50^{* *}$ & $-0,27^{*}$ & $-0,17$ & $-0,05$ & 0,02 & $-0,14$ & $-0,09$ \\
\hline Desaprobación & 1,53 & 1,02 & 0,89 & $0,45^{* *}$ & $-0,17$ & $-0,29^{*}$ & $-0,29^{*}$ & $-0,01$ & $-0,09$ & 0,03 \\
\hline \multicolumn{11}{|l|}{ Mujeres } \\
\hline ECIn & 1,72 & 1,13 & 0,94 & $0,55^{* *}$ & $-0,04$ & $-0,13$ & $-0,17$ & $0,37^{* *}$ & 0,08 & $0,34^{*}$ \\
\hline Amenaza & 1,78 & 1,18 & 0,93 & $0,51^{* *}$ & $-0,03$ & $-0,00$ & $-0,19$ & $0,27^{*}$ & 0,11 & 0,17 \\
\hline Desaprobación & 1,67 & 1,24 & 0,87 & $0,51^{* *}$ & $-0,04$ & $-0,29^{*}$ & $-0,12$ & $0,39^{* *}$ & 0,03 & $0,41^{* *}$ \\
\hline
\end{tabular}

Nota. ECInI = Escala de Competencia Intrasexual a nivel Integrupal. Desaprobación $=$ Desaprobación Normativa, os = Orientación Sociosexual.

** $p<0,001 ; * p<0,05$. 
sexual general $(r=0,47, p<0,001)$, la dimensión conductual de la orientación sociosexual $(r=0,15$, $p=0,025)$ y la dimensión de deseo de la orientación sociosexual ( $r=0,21, p=0,003)$ y presentó una correlación negativa con el valor de potenciales parejas a corto plazo $(r=-0,18, p=0,007)$.

En el caso específico de los hombres, la desaprobación normativa se encontró asociada positivamente con la competencia intrasexual general $(r=0,45, p<0,001)$ y negativamente con el valor asignado a potenciales parejas a largo plazo y corto plazo ( $r s=-0,29, p s=0,002)$. En el caso de las mujeres, la desaprobación normativa de relaciones sexuales intergrupales se encontró correlacionada de manera positiva con la competencia intrasexual $(r=0,51, p<0,001)$, el aspecto conductual de la orientación sociosexual $(r=0,39, p<0,001)$ y el aspecto de deseo de la orientación sociosexual $(r=0,41, p<0,001)$; y de manera negativa con el valor de potenciales parejas a corto plazo $(r=$ $-0,29, p=0,02)$.

\section{Discusión}

Los resultados del Estudio 1 ofrecen primeras evidencias positivas sobre el funcionamiento de la escala de competencia intrasexual a nivel intergrupal en el contexto de la investigación. Aunque su estructura factorial aparenta tener dos subescalas, presenta también un funcionamiento unidimensional con una excelente consistencia interna.

Además, en concordancia con las hipótesis del estudio, las relaciones encontradas con otras variables vinculadas con la selección de pareja proveen evidencias para considerar que el constructo está siendo medido de forma adecuada. En primer lugar, el hecho de que la escala exhibiera una fuerte correlación con la competencia intrasexual, pero dicho valor no fuera excesivamente elevado, proporciona evidencias de validez discriminante. En todos los casos ambas medidas comparten no más del $25 \%$ de la varianza, lo que indica que se trata de medidas de constructos relacionados, pero que no miden exactamente lo mismo. Se asume, junto con Maruyama (1998), que si la correlación hubiera sido muy alta $(0,80$, por ejemplo), se estaría en presencia de un indicador de que ambas medidas miden prácticamente lo mismo (multicolinealidad). Por el contrario, los datos empíricos revelan que la escala de competencia intrasexual a nivel intergrupal parece captar elementos intergrupales que no están contemplados en las escalas de competencia intrasexual.

De igual forma, se encontraron evidencias de validez convergente al observar relaciones significativas entre las puntuaciones de la ECInI con las puntuaciones en medidas de orientación sociosexual y valor asignado a la pareja. Estos datos apoyan la idea de que la medida recoge la compleja relación entre estas variables documentada en investigaciones previas. En concordancia con los planteamientos teóricos, los resultados indican que una persona tenderá a sentir más rivalidad y celos ante personas del exogrupo que lleguen a cortejar a los mujeres del endogrupo, si tiene una mayor inseguridad sobre su valor como pareja, es menos exigente sobre las características de las parejas deseables y se orienta conductualmente a la búsqueda constante de parejas.

Cabe destacar que en este estudio no se encontraron las diferencias esperadas por sexo en la competencia intrasexual a nivel intergrupal que fueron reportadas en estudios anteriores (Klavina \& Buunk, 2013). En este primer estudio no se pueden adelantar explicaciones sobre la ausencia de dichas relaciones, por lo que se hace necesaria una replicación con el fin de determinar si este patrón se mantiene en el contexto de la provincia de Limón o se debe a características idiosincráticas de esta muestra particular.

Adicionalmente, las escalas de validación en este primer estudio solo contemplan aspectos propios de los fenómenos vinculados con la selección de parejas (rivalidad intrasexual, orientación sociosexual, valor como pareja), por lo que no se 
puede asegurar que esta escala capture aspectos importantes y específicos de las relaciones intergrupales, además de los aspectos de competencia intrasexual. Además, el tamaño de la muestra en este estudio no fue lo suficientemente grande como para poder realizar análisis más robustos e inferencias más sólidas.

Por esta razón, se diseñó un segundo estudio cuyo objetivo era proporcionar evidencia empírica que permita tomar decisiones más claras sobre la presencia del segundo factor encontrado en el primer estudio, el cual podría haber emergido como un factor de método. Adicionalmente, el Estudio 2 permitió recopilar más evidencias de validez, incluyendo variables más vinculadas con los aspectos específicos que caracterizan las relaciones intergrupales, como lo son la calidad de contacto intergrupal, el control inhibitorio y la orientación a la dominancia social.

\section{Estudio 2. Estructura y evidencias de validez de la escala en el contexto intergrupal}

\section{Método}

Participantes. Los datos fueron recopilados en una muestra de 335 estudiantes universitarios que participaron de forma voluntaria en el estudio. Las edades de los participantes oscilaban entre los 16 y 30 años $(M=20,79, D E=2,35)$. Un $50,4 \%$ de las participantes eran mujeres. Todos los participantes eran estudiantes de la sede de Limón de la Universidad de Costa Rica.

Instrumentos. El cuestionario incluía el módulo de preguntas sociodemográficas, la ECInI (Klavina \& Buunk, 2013) y la escala de competencia intrasexual general (Buunk \& Fisher, 2009) que se utilizó en el Estudio 1. Además, incluía tres mediciones específicas de variables intergrupales, a saber: la escala de orientación a la dominancia social, la escala de control inhibitorio y un reactivo sobre la calidad de contacto intergrupal.
La escala de orientación hacia la dominancia social (Sidanius \& Pratto, 1994). Las respuestas se recogen en una escala Likert de 1 (totalmente en desacuerdo) a 7 (totalmente de acuerdo). Consta de 16 ítems y posee una consistencia interna adecuada $(\alpha=0,88)$.

Para medir indicadores conductuales del control inhibitorio se utilizó una versión en castellano de la escala BRIEF-A (Behavior Rating Inventory of Executive Function - Adult version) de Roth, Isquith y Gioia (2005), cuyos reactivos se califican en una escala de 0 (nunca) a 6 (Casi siempre). Esta versión consta de 30 ítems y posee una alta consistencia interna $(\alpha=0,92)$.

Finalmente, con el fin de medir la calidad de contacto con personas del exogrupo a los participantes, se les preguntó directamente: "si ha tenido algún contacto o más con personas extranjeras, ¿qué tan negativa o positiva calificaría usted la calidad de contacto que ha tenido con estas personas?". La pregunta se contestaba en una escala de 1 (negativa) a 7 (positiva).

Procedimientos. Al igual que en el Estudio 1 , se contactó a los estudiantes en sus respectivas aulas universitarias en el Recinto de Limón de la Universidad de Costa Rica. Se les explicó el objetivo de la investigación y solicitó llenar voluntariamente el cuestionario. En la portada de este se encontraba el consentimiento informado, siguiendo los lineamientos del comité de ética de la Universidad de Costa Rica. Los y las estudiantes expresaron su acuerdo en participar en la investigación devolviendo el cuestionario completo. Llenar el cuestionario les tomó esta vez 20 minutos. Para los análisis estadísticos se utilizaron los programas SPSS en su versión 16.0 y EQS versión 6.1.

La estimación de los modelos se realizó sobre la base de la matriz de covarianzas de los ítems utilizando el método de máxima verosimilitud (Maximum Likelihood). El ajuste de los modelos fue evaluado con tres índices: el índice de ajuste comparativo (CFI), la raíz cuadrada del error de 
estimación (RMSEA) y el Chi-Cuadrado $\left(\chi^{2}\right)$. Estos índices proveen información acerca de la discrepancia entre la matriz de varianzas/covarianzas propuesta por el modelo teórico y la matriz de varianzas/covarianzas empírica (Hu \& Bentler, 1995). La matriz de varianzas y covarianzas se presenta en la tabla 3.

En general, un modelo se ajusta aceptablemente a los datos si el índice de ajuste comparativo es mayor a 0,90 , la raíz cuadrada del error de aproximación es igual o menor a 0,05 , y el valor Chi-Cuadrado es bajo y no es significativo (Maruyama, 1998). Debido a que el $\chi 2$ por lo general resulta significativo en muestras grandes como estas, aun cuando los modelos se ajusten adecuadamente (Hu \& Bentler, 1995), este índice se utilizó sobre todo para evaluar si las diferencias de ajuste entre los modelos son estadísticamente significativas por medio del test de la diferencia del Chi-Cuadrado $(\Delta \chi 2)$. Complementariamente, se examinaron los coeficientes de asociación entre las variables $(\beta)$ y los índices de modificación (LM Test).

\section{Resultados}

Estructura. Se llevaron a cabo análisis factoriales confirmatorios $(\mathrm{AFC})$ para toda la muestra, así como para hombres y mujeres por separado, y simultáneamente en ambos grupos, utilizando el programa EQS 6.2 (tablas 4 y 5).

El AFC permitió estimar el ajuste de dos modelos: el primer modelo establece que la covariación de los ítems es explicada total y exclusivamente por su relación con un factor latente general denominado Competencia Intrasexual a Nivel Intrasexual; esto debido a que los resultados del análisis exploratorios del Estudio 1 sugerían también una estructura unifactorial de la ECInI.

El segundo modelo predice 2 subfactores covariantes: (I) amenaza, y (II) desaprobación normativa. Aquí, la covariación de los ítems redactados para acceder a cada uno de estos aspectos debería estar explicada exclusivamente por su relación con el respectivo constructo. La relación de los ítems con el factor que no deben medir fue fijada a ce-

Tabla 3

Matriz de varianzas/covarianzas para los items de la escala ECInI

\begin{tabular}{|c|c|c|c|c|c|c|c|c|c|c|c|c|}
\hline & 1 & 2 & 3 & 4 & 5 & 6 & 7 & 8 & 9 & 10 & 11 & 12 \\
\hline 1 & - & 0,427 & 0,376 & 0,329 & 0,265 & 0,258 & 0,399 & 0,392 & 0,401 & 0,318 & 0,397 & 0,382 \\
\hline 2 & & - & 0,400 & 0,421 & 0,388 & 0,429 & 0,455 & 0,360 & 0,396 & 0,334 & 0,419 & 0,528 \\
\hline 3 & & & - & 0,391 & 0,397 & 0,297 & 0,550 & 0,468 & 0,327 & 0,425 & 0,510 & 0,469 \\
\hline 4 & & & & - & 0,494 & 0,444 & 0,518 & 0,474 & 0,398 & 0,416 & 0,386 & 0,510 \\
\hline 5 & & & & & - & 0,543 & 0,504 & 0,501 & 0,415 & 0,358 & 0,376 & 0,535 \\
\hline 6 & & & & & & - & 0,651 & 0,560 & 0,434 & 0,479 & 0,479 & 0,653 \\
\hline 7 & & & & & & & - & 0,761 & 0,475 & 0,532 & 0,593 & 0,703 \\
\hline 8 & & & & & & & & - & 0,486 & 0,423 & 0,506 & 0,670 \\
\hline 9 & & & & & & & & & - & 0,485 & 0,579 & 0,505 \\
\hline 10 & & & & & & & & & & - & 0,802 & 0,494 \\
\hline 11 & & & & & & & & & & & - & 0,562 \\
\hline 12 & & & & & & & & & & & & - \\
\hline
\end{tabular}


ro. Además, este modelo supone que los factores covarían. Esto en virtud de los resultados de la solución rotada del análisis de factores exploratorios del Estudio 1.

Análisis preliminares indicaron la necesidad de establecer la covarianza de los errores de dos ítems que mostraban una relación fuerte en virtud de su contenido semántico. Dichos ítems son:
"Me molesta que un familiar cercano (mi hermano o mi primo) salga con alguien de otro país" y "Me molesta que una familiar cercana (mi hermana o mi prima) salga con alguien de otro país". Todos los modelos presentados incluyen entonces esta especificación.

El modelo que plantea la escala como una estructura unidimensional mostró un ajuste moderado

Tabla 4

Indicadores de bondad de ajuste de los modelos para la ECInI en el Estudio $2(N=335)$

\begin{tabular}{lccccc}
\hline \multicolumn{1}{c}{ Modelo } & gl & $\chi^{2}$ & $\chi^{2} / \mathbf{g l}$ & cfi & RMSEA \\
\hline Un factor & 52 & $283,91^{* *}$ & 5,46 & 0,89 & 0.12 \\
Dos factores & 50 & $230,75^{* *}$ & 4,61 & 0,92 & 0,10 \\
Hombres un factor & 52 & $236,50^{* *}$ & 4,55 & 0,86 & 0,15 \\
Hombres dos factores & 50 & $191,06^{* *}$ & 3,82 & 0,89 & 0,13 \\
Mujeres un factor & 52 & $179,23^{* *}$ & 3,45 & 0,88 & 0,12 \\
Mujeres dos factores & 50 & $163,92^{* *}$ & 3,27 & 0,89 & 0,12 \\
Multigrupo un factor & 116 & $440,82^{* *}$ & 3,80 & 0,86 & 0,13 \\
Multigrupo dos factores & 112 & $376,51^{* *}$ & 3,36 & 0,88 & 0,12 \\
\hline
\end{tabular}

Notas. CFI = Índice de ajuste comparativo, RMSEA = Raíz del error cuadrático medio de aproximación.

**p $p<0,001$

Tabla 5

Solución estandarizada del análisis factorial confirmatorio para el modelo unidimensional de la ECInI en el Estudio $2(N=335)$

\begin{tabular}{cc}
\hline Reactivo & Carga factorial \\
\hline 1 & 0,54 \\
2 & 0,59 \\
3 & 0,83 \\
4 & 0,67 \\
5 & 0,67 \\
6 & 0,80 \\
7 & 0,83 \\
8 & 0,81 \\
9 & 0,69 \\
10 & 0,70 \\
11 & 0,67 \\
12 & 0,68 \\
\hline
\end{tabular}


para el total de la muestra $\left(\chi^{2}=283,91, g l=52, p\right.$ $<0,001 ;$ CFI $=0,89$; RMSEA $=0,12)$. Los resultados indicaron que todos los reactivos cargaron significativamente en un factor general con betas entre 0,46 y 0,85. Los índices de modificación del LM y Wald test no indicaron desajustes significativos en las cargas factoriales.

El modelo de dos factores para toda la muestra se ajustó también de manera relativamente adecuada a los datos $\left(\chi^{2}=230,75, g l=50, p<0,001\right.$; CFI $=0,92 ;$ RMSEA $=0,10)$. Los resultados indicaron que todos los reactivos cargaron significativamente en su respectivo factor y que ambos factores latentes se correlacionan altamente $(r=0,85, p<$ $0,001)$. En este caso, los índices de modificación del LM y Wald test tampoco indicaron desajustes significativos en las cargas factoriales.

En general, los dos modelos presentaron un ajuste muy similar a nivel de la totalidad de la muestra. Sin embargo, la prueba de la diferencia de Chi cuadrado indicó que el modelo bidimensional se ajusta significativamente mejor que el modelo unidimensional $\Delta \chi^{2}=53,16, \mathrm{gl}=2, p<0,001$.

Invariancia de la estructura factorial. Con el fin de estimar la invariancia de la estructura de la escala en hombres y mujeres se ajustaron los dos modelos para hombres y para mujeres por separado y, posteriormente, se llevó a cabo un análisis multigrupo para cada una de las dos estructuras modeladas. En todos los casos solamente las cargas factoriales se fijaron a la igualdad (invariancia débil).

En el caso de los hombres, el modelo unidimensional se ajustó también de manera pobre a los datos $\left(\chi^{2}=236,50, g l=52, p<0,001\right.$; CFI $=0,86$; RMSEA $=0,15)$. Sin embargo, todos los reactivos cargaron significativamente en un factor general con betas entre 0,47 y 0,83 . Los índices de modificación del test de Wald y test LM no indicaron desajustes significativos en las cargas factoriales. El modelo bidimensional también presentó un ajuste relativamente superior a los datos $\left(\chi^{2}=\right.$ $191,06, g l=50, p<0,001$; CFI $=0,89$; RMSEA $=$
$0,13)$. Todos los reactivos cargaron significativamente en sus respectivos factores con betas entre 0,54 y 0,79 en un factor y entre 0,79 y 0,88 en el segundo. Ambos factores se encontraron también altamente correlacionados $(r=0,82, p<0,00)$. Los índices de modificación del test de Wald y test LM tampoco indicaron desajustes significativos en las cargas factoriales. De nuevo, los dos modelos presentaron un ajuste muy similar y la prueba de la diferencia de Chi cuadrado indicó que el modelo bidimensional se ajusta significativamente mejor que el modelo unidimensional $\Delta \chi^{2}=45,44, \mathrm{gl}=$ $2, p<0,001$.

En el caso de las mujeres, el modelo unidimensional se ajustó también de manera moderada a los datos $\left(\chi^{2}=179,23, g l=52, p<0,001\right.$; $\mathrm{CFI}=$ $0,88$; RMSEA $=0,12)$. Todos los reactivos cargaron significativamente en un factor general con betas entre 0,44 y 0,87 y los índices de modificación del test de Wald y test LM no reflejaron desajustes significativos en las cargas factoriales. El modelo bidimensional en mujeres también presentó un ajuste relativamente superior a los datos $\left(\chi^{2}=\right.$ $163,92, g l=50, p<0,001 ; \mathrm{CFI}=0,89$; RMSEA $=$ $0,12)$. Todos los reactivos cargaron significativamente en sus respectivos factores, con betas entre 0,47 y 0,80 en un factor y entre 0,48 y 0,88 en el segundo, y ambos factores latentes presentaron también una alta correlación entre sí $(r=0,89, p$ $<0,001)$. De nuevo, los índices de modificación del test de Wald y test LM tampoco indicaron desajustes significativos en la estructura. Los dos modelos presentaron un ajuste muy similar, pero, al igual que en los casos anteriores, la prueba de la diferencia de Chi cuadrado indicó que el modelo bidimensional se ajusta significativamente mejor que el modelo unidimensional $\Delta \chi^{2}=15,31, \mathrm{gl}=$ $2, p<0,001$.

Los resultados del análisis multigrupo indicaron un ajuste menos adecuado para ambos modelos cuando son ajustados simultáneamente en hombres y mujeres y restringidos a la igualdad $\left(\chi^{2}=440,82\right.$, $g l=116, p<0,001 ; \mathrm{CFI}=0,86$; $\mathrm{RMSEA}=0,13$ para 
el de un factor y $\chi^{2}=376,51, g l=112, p<0,001$; $\mathrm{CFI}=0,88$; $\mathrm{RMSEA}=0,12$ para el de dos factores). Esto sugiere que las estructuras no son completamente invariantes entre hombres y mujeres. Sin embargo, una inspección de los resultados del test LM para cada parámetro no reflejó diferencias significativas en las cargas factoriales entre los hombres y mujeres. Los dos modelos presentaron de nuevo un ajuste muy similar y la prueba de la diferencia de Chi-Cuadrado indicó que el modelo bidimensional se ajusta significativamente mejor que el modelo unidimensional $\Delta \chi 2=66,96, \mathrm{gl}=$ $4, p<0,001$.

En suma, los resultados indican que ambos modelos poseen ajustes muy similares. A pesar de que la prueba de la diferencia de Chi-Cuadrada indica que el modelo bidimensional se ajusta mejor a los datos, las altas correlaciones entre los dos factores apoyan el planteamiento de usar la escala como una medida de un constructo unidimensional. Así, con el principio de la parsimonia en mente, se decidió escoger el modelo unidimensional como el modelo a seguir. Esto tomando en cuenta también que la escala fue desarrollada originalmente de esta forma y que las puntuaciones no difieren mucho si se toman en cuenta como reflejo de una o dos dimensiones.

Consistencia interna. Seguido a esto, se realizaron análisis de consistencia interna solamente para la escala completa. Esta mostró una excelente consistencia interna para toda la muestra $(\alpha=0,91)$ y al dividirla por sexo $(\alpha=0,89$ para mujeres y $\alpha$ $=0,92$ para hombres).

Diferencias por sexo. Si se considera el rango teórico de la escala de 1 a 7 (dadas las opciones de respuesta), la escala general presentó de nuevo un puntaje bajo $(\mathrm{M}=1,96, \mathrm{DE}=1,12)($ tabla 6$)$. La prueba $t$ para muestras independientes no reveló, de nuevo, diferencias significativas por sexo en los niveles de rivalidad expresada ante los miembros del exogrupo $\left(t_{333}=-0,473, p=0,64, d=0,05\right)$.

Evidencias de validez. La matriz de correlaciones simple entre las medidas (tabla 6) evidenció fuertes correlaciones positivas de la ECInI con la escala de competencia intrasexual general $(r$ $=0,55, p<0,001)$ y la escala de orientación hacia la dominancia social $(r=0,47, p<0,001)$, así como correlaciones negativas moderadas con las medidas de calidad de contacto percibida $(r=-0,25, p<0,001)$ y de control inhibitorio $(r=$ $-0,29, p<0,001)$.

\section{Discusión}

Los resultados de este estudio agregan nuevas evidencias sobre el funcionamiento de la ECInI en este contexto. Este segundo estudio evidenció de nuevo la presencia de dos factores altamente correlacionados, como la estructura de mejor ajuste a los datos. Sin embargo, el ajuste de una estructura unifactorial resultó también bastante adecuado para describir la covarianza de los reactivos. Dadas las altas correlaciones entre los factores y en virtud

Tabla 6

Medias, desviaciones estándar, Alfas de Cronbach de la ECInI y sus correlaciones bivariadas con las escalas de validación en el Estudio $2(N=335)$

\begin{tabular}{lccccccc}
\hline & m & SD & $\alpha$ & $\begin{array}{c}\text { Competencia } \\
\text { intrasexual }\end{array}$ & $\begin{array}{c}\text { Dominancia } \\
\text { social }\end{array}$ & $\begin{array}{c}\text { Calidad de } \\
\text { contacto }\end{array}$ & $\begin{array}{c}\text { Control } \\
\text { inhibitorio }\end{array}$ \\
\hline Ambos sexos & 1,96 & 1,12 & 0,91 & $0,55^{* *}$ & $0,47^{* *}$ & $-0,31^{* *}$ & $-0,29^{*}$ \\
Hombres & 1,99 & 1,17 & 0,92 & $0,59^{* *}$ & $0,39^{* *}$ & $-0,27^{* *}$ & $-0,37^{* *}$ \\
Mujeres & 1,93 & 1,07 & 0,89 & $0,51^{* *}$ & $0,59^{* *}$ & $-0,23^{*}$ & $-0,21^{*}$ \\
\hline
\end{tabular}

$* * p<0,001 ; * p<0,05$. 
del principio de parsimonia se recomienda utilizar la escala como una medición de un constructo unidimensional, lo cual concuerda con el diseño original y es respaldado también por la excelente consistencia interna de la escala total.

Las relaciones observadas entre las puntuaciones de la ECInI y los puntajes en las medidas de validación proveen más evidencias para considerar que el constructo está siendo medido de forma adecuada. En primer lugar, el hecho de que siga manteniendo una fuerte correlación con la competencia intrasexual, pero que este valor no sea excesivamente elevado, fortalece las evidencias de validez discriminante: la escala de competencia intrasexual a nivel intergrupal parece captar elementos intergrupales que no están contemplados en las escalas de competencia intrasexual, que serían de mucha utilidad para analizar las implicaciones de la competencia intrasexual en contextos de migración o de relaciones intergrupales cargadas de alta tensión.

Adicionalmente, se encontraron importantes evidencias de validez al observar correlaciones consistentes entre la ECInI y medidas de dominancia social, calidad de contacto y control inhibitorio. Las personas con tendencias a una mayor orientación a la dominancia social, menores niveles de control inhibitorio (un mecanismo importante de control de expresión de estereotipos y prejuicios) y pocas oportunidades de contacto intergrupal de calidad tienden a rivalizar más con personas del exogrupo que lleguen a cortejar a las mujeres del endogrupo. Estos patrones de interrelación concuerdan tanto con las teorías evolucionistas de competencia intrasexual, como con las teorías de la psicología social de las relaciones intergrupales (Allport, 1954; Amodio \& Devine, 2010; Amodio, Devine \& Harmon-Jones, 2007; Devine, Plant, Amodio, Harmon-Jones \& Vance, 2002; Klavina \& Buunk, 2013; Klavina, Buunk \& Park, 2009; Plant \& Devine, 2009; Pettigrew 1998).

Cabe destacar que en este estudio tampoco se encontraron diferencias por sexo en la competencia intrasexual a nivel intergrupal, diferencia que se esperaba encontrar sobre la base de los estudios anteriores (Klavina \& Buunk, 2013). Las razones para no encontrar estas diferencias se hipotetizan en la discusión general.

\section{Discusión general}

Con el propósito de analizar la utilidad de la ECInI (Klavina \& Buunk, 2013) en este contexto se llevaron a cabo dos estudios empíricos en estudiantes universitarios de la provincia de Limón/ CR, Costa Rica. Ambos estudios proporcionaron importantes evidencias de la validez y confiabilidad de las inferencias que se pueden realizar con las puntuaciones de la escala para estudiar los fenómenos de la selección de pajera y la rivalidad intrasexual en contextos intergrupales.

Los resultados no son concluyentes pero, de igual forma, se podría recomendar la utilización de la escala como una medida de un constructo unidimensional que parece captar las reacciones de hombres y mujeres ante la presencia de rivales extranjeros en la selección de pareja. Por otro lado, se recomienda la realización de más estudios que busquen confirmar la estructura factorial de esta escala con el fin de determinar un adecuado uso de la escala unidimensional o bifactorial.

Quizá el hallazgo más desconcertante de los estudios acá presentados es la ausencia de diferencias significativas entre hombres y mujeres a nivel de la rivalidad y la competencia intrasexual intergrupal. Diferencias que han sido usuales en las demás investigaciones empíricas sobre el tema (Buunk, Massar \& Dijkstra, 2007; Buunk \& Fisher, 2009; Dijkstra \& Buunk, 2002). En los estudios acá presentados los niveles de competencia intrasexual-intergrupal de hombres y mujeres resultaron muy similares. Esto puede obedecer a diversas razones.

Una primera razón puede estar asociadacon las particularidades del contexto social donde fue realizada la investigación. La provincia de Limón es 
una zona costera, un puerto, que se caracteriza por ser un contexto pluricultural donde habitan grupos de diferentes procedencias etnoculturales (afrocostarricenses, panameños, nicaragüenses, mestizos, caucásicos, etc.) (Instituto Nacional de Estadísticas y Censos, 2011) y en dónde la migración es común, lo cual propicia un mayor contacto entre los diferentes grupos y la promoción de identidades comunes en sus habitantes, independientemente de las diferencias individuales y las nacionalidades. Esta particularidad pudo haber contribuido a que no se observaran diferencias en las respuestas de hombres y mujeres ante extranjeros; sin embargo, esta es una hipótesis que habría que confirmar con estudios posteriores.

En otras palabras, los grupos extranjeros en este contexto no resultan tan amenazantes para los habitantes de Limón porque están habituados a su presencia. Las respuestas típicas ante la amenaza que despliegan hombres y mujeres pueden entonces haberse obscurecido por esta razón. De hecho, los niveles reportados de celos y rivalidad ante extranjeros tanto en hombres como mujeres fueron muy bajos (menos de 2 puntos en una escala de 1 a 7 en ambos estudios). Es probable que en otros contextos sociales, caracterizados por menor diversidad cultural, las diferencias entre hombres y mujeres emerjan más claramente. Esta hipótesis deberá ser contrastada en futuros estudios en zonas de diversa composición étnica, con el fin de comparar la rivalidad intrasexual-intergrupal entre hombres y mujeres en diversos contextos.

En esta misma línea, es importante tomar en cuenta que en otros estudios del campo de la psicología evolucionista (Buunk, Castro-Solano, Zurriaga \& González, 2011) se han encontrado diferencias entre los contextos latinoamericanos y europeos, aspecto que puede señalar diferencias culturales en la competencia intrasexual.

Una segunda razón, de orden técnico, puede estar asociada con la ausencia de contextualización clara a la hora de aplicar las medidas. Comúnmente, las medidas que intentan captar reacciones ante la presencia de rivales introducen en sus instrucciones situaciones evocadoras de competencia intrasexual (Johnson \& Jacobson, 2005). En los cuestionarios acá utilizados, tal situación evocadora de competencia estaba ausente. A lo mejor, si se introduce previamente al participante en un contexto de competencia intergrupal se logre evocar mayor rivalidad hacia los miembros del exogrupo (Allport, 1954; Johnson \& Jacobson, 2005; Pettigrew, 1988) y en un contexto de este tipo las diferencias entre hombres y mujeres pueden emerger más claramente. Futuros estudios podrían abocarse a contrastar esta hipótesis.

A pesar de estas limitaciones, la escala de competencia intrasexual a nivel intergrupal posee propiedades psicométricas adecuadas para captar aspectos de las relaciones sociales que no se pueden captar ni con las escalas de competencia intrasexual ni con las medidas típicas de relaciones intergrupales existentes. Estos aspectos, hasta ahora no medidos, pueden arrojar luz para comprender la expresión de estereotipos y prejuicios en contextos pluriculturales desde la perspectiva de la psicología evolucionaria y la psicología social.

Muchas de las respuestas agresivas ante la migración o la diversidad cultural (como el prejuicio, los estereotipos negativos y la discriminación) responden a mecanismos cognitivos, afectivos y motivacionales fuertemente arraigados en nuestra especie, que en su momento fueron necesarios para la supervivencia en la competencia intergrupal sobre los recursos. Medidas que permitan captar estas realidades resultan muy útiles para conocer estos fenómenos más a fondo y transformarlos.

\section{Referencias}

Allport, G. (1954). The nature of prejudice. Reading, MA: Perseus Book Publishing.

Amodio, D. M., \& Devine, P. G. (2010). Control in the regulation of intergroup bias. In R. R. Hassin, K. N. Ochsner \& Y. Trope (Eds.), Self-control 
in society, mind, and brain (pp. 49-75). Oxford ; N.Y.: Oxford University Press.

Buss, D. M. (2006). Strategies of human mating. Psychological Topics, 15, 239-260.

Buunk, A. P., Castro-Solano, A., Zurriaga, R., \& González, P. (2011). Gender Differences in the Jealousy-Evoking Effect of Rival Characteristics: A Study in Spain and Argentina. Journal of Cross-Cultural Psychology, 42(3), 323-339. doi:10.1177/0022022111403664

Buunk, A. P., \& Fisher, M. (2009). Individual differences in intrasexual competition. Journal of Evolutionary Psychology, 7, 37-48.

Buunk, A. P., Massar, K., \& Dijkstra, P. (2007). A social cognitive evolutionary approach to jealousy: The automatic evaluation of one's romantic rivals. En Forgas, J. P., Haselton, M. G. \& von Hippel, W. (Eds.), Evolution and the social mind: evolutionary psychology and social cognition (pp. 213-228). New York: Psychology Press.

Buunk, A. P., Park, J. H., \& Duncan, L. A. (2010). Cultural variation in parental influence on mate choice. Cross-Cultural Research, 44, 23-40.

Dijkstra, P., \& Buunk, B. P. (2002). Sex differences in jealousy-evoking effect of rival characteristics. European Journal of Social Psychology, $32,829-852$.

Fisher, M., Tran, U., \& Voracek, M. (2008). The Influence of Relationship Status, Mate Seeking, and Sex on Intrasexual Competition. The Journal of Social Psychology, 148, 493-508.

Guadagnoli, E., \& Velicer, W. F. (1988). Relation of sample size to the stability of component patterns. Psychological Bulletin, 103, 265-275.

Hoe, S. L. (2008). Issues and procedures in adopting structural equation modeling technique. Journal of Applied Quantitative Methods, 3(1), 76-83.

Hu, L., \& Bentler, P. M. (1995). Evaluating model fit. En R. Hoyle (Ed.). Structural equation modeling: Issues, concepts, and applications (pp. 76-99). Newbury Park, C.A.: Sage.
Instituto Nacional de Estadísticas y Censos (2011). Censo Nacional de Población y Vivienda, 2011.

Johnson, B. R., \& Jacobson, C. K. (2005). Contact in context: An examination of social settings on whites' attitudes toward interracial marriage. Social Psychology Quarterly; 68, 387-399.

Kirsner, B. R., Figueredo, A. J., \& Jacobs, W. J. (2003) Self, Friends and Lovers: structural relations among Beck Depression Inventory scores and perceived mate values. Journal of Affective Dissorders, 75(2), 131-148.

Klavina, L., \& Buunk, A. (2013). Intergroup intrasexual competition: Reactions towards outgroup members as romantic rivals. Journal of Evolutionary Psychology, 11, 93-120.

Klavina, L., Buunk, A. P., \& Park, J. P. (2009). Intergroup jealousy: Effects of perceived group characteristics and intrasexual competition between groups. In Hogh-Olesen, H., Tonnesvang, J. \& Bertelsen, P. (Eds.), Human Characteristics: Evolutionary Perspectives on Human Mind and Kind (pp. 382-397). UK: Cambridge Scholars Publishing.

Maruyama, G. (1998). Basics of Structural Equation Modeling. Thousand Oaks CA: Sage.

Morrison, K. R., Fast, N. J., \& Ybarra, O. (2009). Group status, perceptions of threat, and support for social inequality. Journal of Experimental Social Psychology, 45(1), 204-210. doi:10.1016/j.jesp.2008.09.004

Oldmeadow, J., \& Fiske, S. T. (2007). System-justifying ideologies moderate status $=$ competence stereotypes: roles for belief in a just world and social dominance orientation. European Journal of Social Psychology, 37(6), 1135-1148. doi:10.1002/ejsp.428.

Penke, L. (2010). The revised Sociosexual Orientation Inventory. En T. D. Fisher, C. M. Davis, W. L. Yarber \& S. L. Davis (Eds.), Handbook of sexuality-related measures $3^{\text {rd }}$ ed., (pp. 622624). UK: Taylor \& Francis.

Pettigrew, T. F. (1998). Intergroup contact theory. Annual Review of Psychology, 49, 65-85. 
Plant, E. A., \& Devine, P. G. (2009). The active control of prejudice: Unpacking the intentions guiding control efforts. Journal of Personality and Social Psychology, 96(3), 640-652. doi:10.1037/a0012960

Roth, R. M., Isquith, P. K., \& Gioia, G.BA. (2005). Behavioral rating inventory of executive function-Adult version, Lutz, FL: Psychological Assessment Resources, Inc.

Sandoval, C. (2002). Otros amenazantes: los nicaragüenses y la formación de identidades nacionales en Costa Rica. San José, CR: Editorial de la Universidad de Costa Rica.
Sheng, Y., \& Sheng, Z. (2012). Is coefficient alpha robust to non-normal data? Front. Psychology 3, doi: 10.3389/fpsyg.2012.00034.

Sidanius, J., \& Pratto, F. (1999). Social dominance: An intergroup theory of social hierarchy and oppression. Cambridge: Cambridge University Press.

Smith-Castro, V., Araya, M., \& Peña, L. (2009). ¿Costa Rica solidaria?: actitudes hacia la migración y los migrantes. Revista Digital de la Maestría en Ciencias Penales de la Universidad de Costa Rica, 1, 519-533.
Recibido: 06 de enero de 2015

Aprobado: 16 de diciembre de 2016 